\title{
Efeitos da manipulação do estímulo visual e da intenção na oscilação postural de idosas
}

\author{
Effects of visual stimulus manipulation and of intention on postural \\ oscillation of elderly women
}

\author{
Andrei Guilherme Lopes ${ }^{1}$, Milena Razuk ${ }^{2}$, José Angelo Barela ${ }^{3}$
}

Estudo desenvolvido no

Laboratório para Estudos do Movimento, Depto. de Educação Física do IB/Unesp Instituto de Biociências da Universidade Estadual Paulista, campus de Rio Claro, Rio Claro, SP, Brasil

1 Doutorando no Depto. de Educação Física do IB/Unesp

2 Graduanda no IB/Unesp

3 Prof. Dr. do Instituto de Ciências da Atividade Física e Esporte da Universidade Cruzeiro do Sul, São Paulo, SP

\section{ENDEREÇO PARA}

CORRESPONDÊNCIA

José Ângelo Barela Instituto de Ciências da Atividade Física e Esporte / Unicsul

R. Galvão Bueno 868 01506-000 São Paulo SP e-mail: jose.barela@cruzeirodosul.edu.br

Este estudo contou com bolsas da Capes - Coordenação de Aperfeiçoameto de Pessoal de Nível Superior - e do CNPq Conselho Nacional de Desenvolvimento Científico e Tecnológico. para os autores ${ }^{1}$ $\mathrm{e}^{2}$, respectivamente.

Apresentação out. 2008

ACEITO PARA PUBLICAÇÃo mar. 2009
Resumo: Este estudo investigou a influência de características do estímulo visual e o efeito da intenção nas respostas do controle postural frente à manipulação visual de adultas idosas. As 20 participantes permaneceram em pé em uma sala móvel durante sete tentativas com duração de 1 minuto cada, olhando para um alvo fixo, medindo-se sua oscilação corporal. Na primeira tentativa não houve qualquer movimento da sala, porém a partir da segunda a sala foi movimentada no sentido ântero-posterior. Para dez participantes, a velocidade de pico da movimentação foi de $0,6 \mathrm{~cm} / \mathrm{s}$ e, para as demais, de $1,0 \mathrm{~cm} / \mathrm{s}$. A partir da quinta tentativa, as participantes foram informadas do movimento da sala e orientadas a resistir à movimentação. Os resultados indicam que a oscilação corporal das idosas é induzida pelo movimento da sala móvel. Intenção e alteração da característica do estímulo visual reduzem a influência da informação visual na oscilação corporal, mas a manipulação de propriedade do estímulo (neste caso, velocidade), é menos efetiva que a intenção. Essa maior dependência da intenção para alterar a influência de um estímulo sensorial no controle postural indica que o funcionamento do sistema de controle postural em idosos não possibilita ajustes "automáticos" de respostas posturais frente a pequenas variações das condições ambientais. linformações sobre tais variações podem ser direcionadas de forma a compensar essa diferença.

Descritores: Idoso; Mulheres; Percepção visual; Postura

ABSTRACT:This study examined the influence of both visual stimulus features and intention on postural control responses due to visual manipulation of elderly women. Twenty participants stood upright inside a moving room, for seven trials for $1 \mathrm{~min}$ apiece, staring at a fixed target, their body sway being measured. The room was not moved during the first trial, but from the second trial on, it was continuously oscillated back and forward. For ten participants, the moving room oscillated with peak velocity of $0.6 \mathrm{~cm} / \mathrm{s}$ and for the others, with peak velocity of $1.0 \mathrm{~cm} / \mathrm{s}$. From the fifth trial on, participants were informed about the movement of the room and instructed to resist to its influence. Results show that body sway is induced by visual manipulation in elderly adults. Intention and change in visual stimulus lead to less influence of the visual information on body sway, but changes in the stimulus properties (velocity, for that matter), is less effective than intention. This higher dependency on intention for changing sensory influence on postural control suggests that postural control in elderly adults does not benefit from "automatic" postural responses due to small environmental changes. Tips and information about such kind of environmental changes should be used in order to make up for such difference in automatic adjustments observed in the elderly.

KEy words: Aged; Posture; Visual perception; Women 


\section{INTRODUÇÃO}

O controle postural envolve um intrincado relacionamento entre informação sensorial e comandos motores para garantir a estabilidade e a manutenção de uma posição corporal desejada. Recentemente, diversos estudos têm examinado essa relação em adultos idosos, para identificar possíveis alterações no funcionamento do sistema de controle postural nessa população ${ }^{1-3}$. Entretanto, ainda há muito a ser entendido sobre as alterações funcionais no controle postural decorrentes do processo de envelhecimento.

Durante a manutenção da postura em pé, adultos idosos oscilam mais que adultos jovens $\mathrm{s}^{4-6}$ e apresentam uma menor e menos controlada região de excursão do centro de pressão ${ }^{7}$, sendo que essas diferenças são ainda mais marcantes em situações com maior demanda do controle postural ${ }^{1,8}$. A necessidade de desvendar e entender os mecanismos responsáveis por essas alterações no controle postural de idosos é ainda mais premente dada a maior ocorrência de quedas em adultos idosos 9,10 e os inúmeros transtornos que enfrentam, decorrentes dessas quedas. Apesar dos inúmeros esforços em identificar características comportamentais ${ }^{11}$, indicadores ${ }^{12,13}$ e sugerir atividades de intervenção ${ }^{14}$, a ocorrência do elevado número de quedas, o estabelecimento de medidas de prevenção e o entendimento das alterações no funcionamento do controle postural em idosos ainda não foram totalmente estabelecidos ${ }^{15}$.

Horak e McPherson ${ }^{16}$ sugeriram que o sistema de controle postural tem como objetivos comportamentais a orientação e o equilíbrio postural, que são alcançados por uma complexa e dinâmica relação entre informação sensorial e ação motora. Nesse caso, estímulos provenientes dos diversos canais sensoriais são integrados pelo sistema nervoso central, possibilitando a formação do quadro de referência utilizado para identificar o relacionamento entre os segmentos e a orientação destes em relação ao ambiente ${ }^{17}$. Com base nesse quadro de referência, atividade muscular específica é desencadeada, buscando manter ou alcançar a orientação e o equilíbrio postural desejado. Nas situações em que essa dependência mútua entre informação sensorial e ação motora é manifestada de forma regular, ocorre a formação do ciclo percepção-ação ${ }^{18}$, que pode ser examinado para inferir como a informação sensorial é utilizada pelo sistema de controle postural.

A influência de informação sensorial no controle postural de idosos tem despertado interesse de diversos estudiosos. Wade e colaboradores ${ }^{6}$ foram os pioneiros a examinar a influência da manipulação da informação visual na oscilação corporal de adultos idosos, utilizando movimentos discretos de uma sala móvel, e observaram maior oscilação corporal de adultos idosos que jovens. Resultados similares foram observados quando idosos não só foram mais susceptíveis ao movimento discreto de uma sala móvel, mas também apresentaram uma relacão mais forte entre o movimento da sala e a oscilação corporal desencadeada na condição de movimentos oscilatórios e contínuos da sala ${ }^{3}$. Esses resultados sugerem que, embora idosos apresentem menor acuidade e sensibilidade ao contraste visual ${ }^{19,20}$, essas alterações não são suficientes para evitar o uso de informação visual pelo sistema de controle postural.

Interessante, entretanto, é que os idosos não conseguiram detectar manipulação da informação visual, enquanto adultos jovens foram capazes $^{21}$. Assim, embora o sistema de controle postural de idosos seja capaz de utilizar informação visual para manter e controlar a posição em pé, o uso da informação visual parece não ser tão refinado quanto em adultos jovens. Mais ainda, a influência da manipulação da informação visual na postura em pé em adultos é alterada quando é apresentada informação sobre a referida manipulação ${ }^{22}$ ou solicitação para evitar sua influência ${ }^{23,24}$. Infelizmente, informações sobre os efeitos de manipulação do estímulo visual e da solicitação de evitar sua influência não foram ainda examinadas em idosos. Tais informações podem ser importantes para entender aspectos do funcionamento do sistema de controle postural de idosos. Entretanto, mais importante é compreender como fornecer e manipular informação sensorial a idosos nas situações do dia-a-dia e/ou no delineamento de protocolos de intervenção. Assim, o objetivo deste estudo foi examinar a influência das características do estímulo visual e o efeito da intenção nas respostas do controle postural de idosos frente à manipulação da informação visual.

\section{METODOLOGIA}

Participaram deste estudo 20 idosas $(62,5 \pm 2,12$ anos) fisicamente ativas e sem lesões ou distúrbios musculoesqueléticos conhecidos ou alterações neurológicas que pudessem afetar a capacidade de manter o equilíbrio. A opção por apenas mulheres visou maior homegeneidade da amostra. Elas foram distribuídas aleatoriamente em dois grupos experimentais: "velocidade baixa" (VB) ( $n=10 ; 64,8 \pm 6,9$ anos), expostas a um estímulo visual com velocidade pico de $0,6 \mathrm{~cm} / \mathrm{s}$; e "velocidade alta" (VA) ( $n=10 ; 61 \pm 3,6$ anos), expostas a um estímulo visual com pico de velocidade de $1,0 \mathrm{~cm} / \mathrm{s}$. Todas as participantes compareceram uma única vez ao Laboratório para Estudos do Movimento do Departamento de Educação Física, Instituto de Biociências, Unesp Rio Claro e, antes de participarem do estudo, assinaram um termo de livre consentimento, aprovado pelo Comitê de Ética da instituição.

\section{Procedimentos}

O experimento foi desenvolvido em uma sala móvel formada por três paredes e um teto montados sobre rodas, que deslizam no sentido ântero-posterior sobre trilhos, de maneira independente do chão. O teto foi pintado de branco e as paredes de preto e branco com faixas verticais de $20 \mathrm{~cm}$ de largura. Uma lâmpada fluorescente foi fixada no centro do teto da sala para iluminação interna. Um programa específico (Compumotor - Motion Architect for Windows) controlava o movimento da sala, por um sistema de servo-mecanismo (Compumotor). Os dados sobre os deslocamentos de oscilação corporal e da sala móvel, na freqüência de $100 \mathrm{~Hz}$, foram adquiridos por um sistema de análise tridimensional de movimento (Optotrak 3020 - Northern Digital Inc.), com um emissor infravermelho fixado entre as escápulas das participantes e outro na parede frontal da sala. 
As participantes foram instruídas a permanecer na postura em pé e paradas dentro da sala móvel, descalças e com os braços relaxados ao lado do corpo, sendo realizadas sete tentativas, cada uma com 60 s de duração. Na primeira tentativa, a sala permaneceu sem movimento, porém nas outras seis a sala foi movimentada na direção ântero-posterior, com movimentos oscilatórios na freqüência de $0,2 \mathrm{~Hz}$. Nas primeiras três tentativas em que a sala se movimentou, as participantes foram orientadas a olhar fixamente para um alvo preso à parede frontal fixa, na altura dos olhos a uma distância de $1 \mathrm{~m}$, e não foram informadas sobre o movimento da sala (condição normal). Nas três tentativas subseqüentes, as participantes foram informadas sobre o movimento da sala $e$ orientadas a, além de fixar o olhar no alvo como nas tentativas anteriores, resistir ao movimento da sala (condição resistida). A sala foi movimentada em diferentes velocidades e amplitudes para cada grupo. O grupo VB foi exposto ao movimento com pico constante de velocidade de $0,6 \mathrm{~cm} / \mathrm{s}$ e amplitude de 1,2 $\mathrm{cm}$. O grupo VA foi exposto ao movimento da sala com pico constante de velocidade de $1,0 \mathrm{~cm} / \mathrm{s}$ e amplitude de $1,9 \mathrm{~cm}$

\section{Análise dos dados}

Em todas as tentativas, foi obtida a amplitude média de oscilação (AMO) como medida da magnitude total da oscilação corporal na direção ântero-posterior. A AMO foi calculada obtendo o desvio padrão do sinal da oscilação corporal depois que um polinômio linear e a média da posição da oscilação corporal foram subtraídos dos valores da respectiva tentativa.

Para as tentativas em que a sala foi movimentada, a análise da relação entre o deslocamento da sala e a oscilação corporal foi realizada apenas na direção ântero-posterior, pois a manipulação da informação visual ocorreu nessa direção. Para analisar a relação temporal e a influência da informação visual na oscilação corporal em ambas as condições, normal ou resistida, quatro variáveis foram utilizadas: coerência, ganho, fase e amplitude espectral na freqüência do estímulo (stimulus frequency spectrum amplitude - SFSA). Coerência indica a força da relação entre o estímulo visual e a oscilação corporal: valores de coerência próximos de 1 indicam forte dependência e, inversamente, valores próximos de 0 revelam fraca ou nenhuma dependência entre o estímulo visual e a oscilação corporal. O ganho infere o quanto a manipulação do estímulo visual (ou seja, a oscilação da sala) induz oscilação corporal; é calculado como a razão entre as amplitudes dos espectros da oscilação corporal e da oscilação da sala. Valores de ganho menores ou maiores do que 1 indicam que a amplitude do espectro de oscilação corporal foi menor ou maior do que a amplitude de oscilação da sala, respectivamente. Tanto a coerência quanto o ganho foram calculados na freqüência em que o estímulo visual foi manipulado, neste caso, na freqüência de 0,2 Hz.

A variável fase foi utilizada para verificar a relação temporal entre a oscilação corporal e o estímulo visual. Valores de fase maiores ou menores do que zero indicam que a oscilação corporal está adiantada ou atrasada em relação à oscilação da sala móvel, respectivamente. Valores de fase iguais a zero indicam que não há diferença temporal entre a oscilação corporal e o movimento da sala. Finalmente, a SFSA indica a magnitude espectral da oscilação corporal na freqüência de oscilação da sala $(0,2$ $\mathrm{Hz}$ ). Nesse caso, valores maiores indicam que a manipulação da informação visual influenciou mais a oscilação corporal das participantes do que valores menores de SFSA. Todas as análises foram realizadas por rotinas específicas, utilizando o programa Matlab (Math Works Inc., v. 7.0). Nenhuma normalização referente à oscilação corporal foi realizada, pois os grupos eram homogêneos quanto à estatura.

Uma análise de multivariância (Manova) e duas análises de variância (Anovas), tendo grupo (VB e VA) e condição (normal e resistida) como fatores, esta última tratada como medidas repetidas, foram utilizadas para analisar a influência da manipulação da informação visual na oscilação corporal das participantes. As variáveis dependentes da Manova foram coerência e ganho e da Anova foram fase e SFSA. Uma ter- ceira Anova, tendo grupo (VB e VA) e condição (sem movimento da sala, normal e resistida) como fatores (este último também tratado como medidas repetidas) e variável dependente a amplitude média de oscilação, foi utilizada para verificar a magnitude de oscilação corporal dos participantes. Quando aplicáveis, análises univariadas e teste post hoc de Tukey foram utilizados. O nível de significância foi de 0,05 para todas as análises e os testes estatísticos foram feitos utilizando o programa SPSS (v. 10.0 for Windows).

\section{RESULTADOS}

A manipulação da informação visual, decorrente da oscilação da sala móvel, influenciou a oscilação corporal dos participantes de ambos os grupos e em ambas as condições. A análise feita mostra que a oscilação corporal acoplou-se ao movimento da sala em todas as condições. Gráficos espectrais mostram picos definidos para oscilação corporal e da sala, ao redor de $0,2 \mathrm{~Hz}$, indicando que ambos oscilaram ao redor dessa freqüência. Na condição resistida, o grupo VA apresentou um pico de oscilação corporal menor, comparado ao grupo VB na mesma condição.

O Gráfico 1 apresenta os valores de coerência e ganho para os grupos VB e VA nas condições normal e resistida. A análise multivariada revelou efeito significante para grupo [Wilks' Lambda $=0,53 ; \mathrm{F}(2,17)=7,32 ; p<0,01]$, condição [Wilks' Lambda $=0,47$; $F(2,17)=9,95 ; p<0,005]$ e interação grupo e condição [Wilks' Lambda $=0,62$; $\mathrm{F}(2,17)=5,21 ; p<0,05]$. A análise univariada para grupo indicou diferença apenas para ganho $[F(1,18)=13,69$; $p<0,005]$, com o grupo VB apresentando valores maiores de ganho do que o grupo VA. Para condição, indicou diferença para coerência $[F(1,18)=17,18$; $p<0,005]$ e ganho $[F(1,18)=19,98$; $p<0,001$ ], sendo que para ambas as variáveis os valores na condição resistida foram menores do que na condição normal. A análise univariada para interação grupo e condição não revelou qualquer diferença significativa nas variáveis coerência e ganho.

O Gráfico 2 apresenta os valores de 

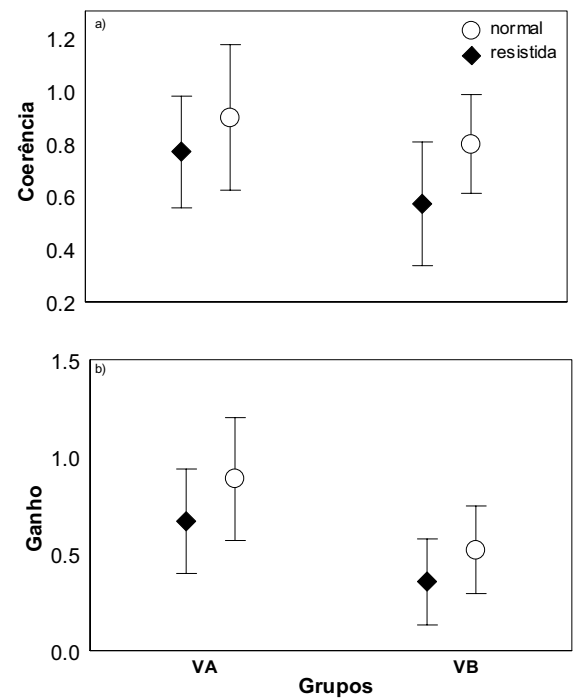

Gráfico 1 Valores médios de coerência (a) e ganho (b) para os grupos de velocidade baixa (VB) e velocidade alta (VA) nas condições normal e resistida

fase para os grupos VB e VA nas condições normal e resistida. A Anova não revelou qualquer diferença significante para grupo $[\mathrm{F}(1,18)=0,68 ; p=0,41]$, condição $[F(1,18)=0,01 ; p=0,91]$ nem interação entre grupo e condição $[F(1,18)=0,002 ; p=0,96]$. De forma geral, os resultados mostram que os participantes oscilaram em fase com o movimento da sala.

O Gráfico 3 apresenta os valores de SFSA para os grupos VB e VA nas condições normal e resistida. A anãlise revelou diferença significante para condição $[F(1,18)=20,39 ; p<0,001]$, porém não

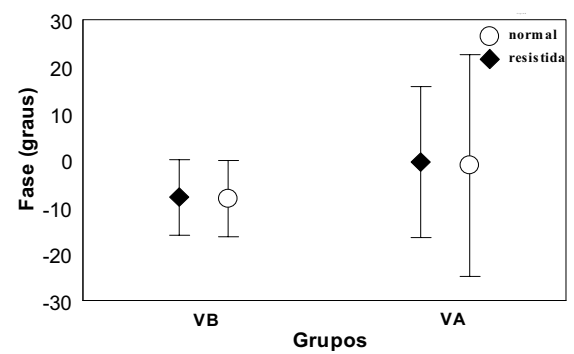

Gráfico 2 Valores médios de fase para os grupos de velocidade baixa (VB) e de velocidade alta (VA) nas condições normal e resistida

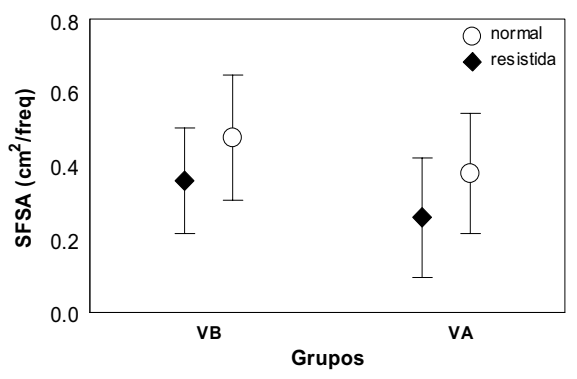

Gráfico 3 Valores médios da amplitude espectral na freqüência do estímulo (SFSA) para os grupos de velocidade baixa (VB) e de velocidade alta (VA) nas condições normal e resistida

revelou efeito para grupo $[F(1,18)=2,77$; $p=0,11]$ nem interação grupo e condição $[F(1,18)=0,002 ; p=0,96]$. Os valores de SFSA indicaram que a oscilação corporal desencadeada pelo movimento da sala foi menor na condição resistida do que na condição normal.

Finalmente, o Grãfico 4 apresenta os valores da amplitude média de oscilação (AMO) dos grupos VB e VA nas condições sem movimento, normal e resistida. A análise revelou diferença significante para condição $[F(2,17)=4,66 ; p<0,05] \mathrm{e}$ interação grupo e condição $[F(2,17)=5,50$; $p<0,05]$, porém nenhum efeito para grupo $[\mathrm{F}(1,18)=0,14 ; p=0,70]$. Testes post hoc indicaram apenas que as participantes do grupo VA oscilaram mais na condição normal, em que a sala foi movimentada e as participantes não sabiam do movimento, do que quando a sala não foi movimentada.

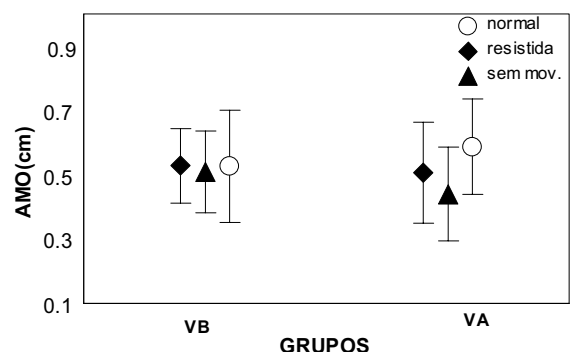

Gráfico 4 Valores da amplitude média de oscilação (AMO) dos grupos de velocidade baixa (VB) e de velocidade alta (VA) nas condições sem movimento, normal e resistida
DISCUSSÃO E CONCLUSÃO

Os resultados mostraram que a oscilação corporal de idosos é induzida pelo movimento de uma sala móvel. E, ainda, que a intenção e a alteração da característica do estímulo visual reduzem a influência da informação visual na oscilação corporal, porém a manipulação do estímulo é menos efetiva que a intenção. Essa maior dependência da intenção para alterar a influência de um estímulo sensorial na oscilação corporal é um fator complicador para o funcionamento do sistema de controle postural em idosos.

Oscilação corporal semelhante em idosos, desencadeada pela manipulação da informação visual, tem sido verificada em diversos estudos, tanto com movimentos discretos ${ }^{3,6}$ quanto com movimentos contínuos e oscilatórios da sala móvel l,3,21 $^{2,3}$ e, portanto, essa constatação não é nova. Entretanto, é importante salientar que idosos acoplam sua oscilação à da sala, mesmo que o movimento da sala envolva oscilações com amplitudes pequenas $(1 \mathrm{~cm})$, como neste e em outros casos ${ }^{3}$. Assim, mesmo que o processo de enveIhecimento implique alterações na acuidade e sensibilidade ao contraste $\mathrm{e}^{19,20}$, essas alterações não são suficientes para evitar ou até mesmo prejudicar o uso de informação visual pelo sistema de controle postural. Os valores de ganho e coerência observados neste estudo, indicando a influência e a relação entre o movimento da sala e a oscilação corporal, respectivamente, são similares aos de outros estudos, que utilizaram manipulação visual com parâmetros idênticos e que não observaram diferença entre adultos jovens e idosos ${ }^{3}$. $\mathrm{Na}$ realidade, em algumas situações em que a estabilidade postural é ameaçada de forma mais contundente, idosos apresentaram oscilação corporal maior que adultos jovens ${ }^{3,6}$. Novamente, qualquer alteração do sistema visual decorrente do envelhecimento não compromete o uso dos estímulos provenientes desse canal sensorial para controlar a oscilação corporal.

A magnitude da influência da manipulação visual pode ser alterada quando adultos idosos são informados sobre 
o movimento da sala e solicitados a resistir à influência dessa manipulação. Essa redução na influência da manipulação visual havia sido observada em adultos jovens ${ }^{22,23,25}$ e em crianças $^{24}$ e a constatação de que adultos idosos também são capazes de reduzir essa influência é importante para o entendimento do funcionamento do sistema de controle postural dessa população. Vale lembrar que, no presente e em outros estudos ${ }^{22,25}$, participantes não foram capazes de discriminar conscientemente que a informação visual estava sendo manipulada. Assim, o sistema de controle postural acopla-se ao estímulo visual de forma "automática"23 ou que reflita a dinâmica de relacionamento entre informação sensorial e ação motora ${ }^{22,25}$. Entretanto, essa dinâmica pode ser alterada quando adultos jovens são informados por outrem $^{22}$ ou solicitados a resistir à manipulação ${ }^{23}$. Os resultados do presente estudo indicam que essa alteração também ocorre no caso de idosos.

Embora idosos consigam reduzir a influência da informação visual quando são informados sobre a manipulação, a alteração da magnitude do estímulo visual quase não induz qualquer alteração na influência visual. Nesse caso, com o aumento da amplitude e velocidade do movimento da sala, apenas a variável ganho foi reduzida, indicando que idosos não acompanharam, com aumento da oscilação correspondente, o aumento do estímulo visual. Esse resultado é diferente de outros recentemente obtidos com adultos jovens 25 , quando os mesmos foram capazes de reduzir o efeito da manipulação visual. Portanto, adultos idosos parecem não conseguir discriminar pequenas variações dos estímulos sensoriais disponíveis no ambiente e modular as respostas posturais da mesma forma como adultos jovens o fazem.

\section{Controle postural em adultos idosos: implicações}

A constatação de que a manipulação de propriedades do estímulo sensorial provoca uma pequena alteração na oscilação corporal de idosos indica alterações importantes no funcionamento do sistema de controle postural dessa po- pulação. Primeiro, idosos são sensíveis às pequenas variações do estímulo visual, mas, comparando com dados de estudos prévios ${ }^{25}$, as respostas não são tão bem calibradas quanto as observadas em adultos jovens. Segundo, essa diferença no uso de informação sensorial no controle dos movimentos pode ser compensada por informação fornecida explicitamente aos idosos.

A relação entre estímulo sensorial e ação motora correspondente no controle postural não depende de atuação consciente da pessoa, sendo automáti$\mathrm{Ca}^{23}$ mesmo em bebês com poucos dias ${ }^{26}$ ou meses $^{27}$ de idade, e sugerida como inata da espécie humana ${ }^{28}$. A independência de atuação consciente que governe esssa relação entre informação sensorial e ação motora permite uma funcionalidade muito importante ao sistema de controle postural, pois não há necessidade de direcionar atenção e controle específico para esse tipo de atividade. Apesar da funcionalidade de não ter de dividir atenção com mecanismos desse tipo de controle, o sistema precisa ainda garantir adaptabilidade, de forma que variações no estímulo sensorial sejam também incorporadas no controle de ação motora. Recentemente, estudos têm buscado investigar e entender a dinâmica desses mecanismos, sugerindo um processo de repesagem sensori$\mathrm{a}^{24,29,30}$. Infelizmente, apenas alguns estudos examinaram esses aspectos em ido$\mathrm{SOS}^{2}$; e indicaram que idosos apresentam a capacidade de alterar as influências de estímulos sensoriais no controle da postura ereta, sendo mais influenciados por tais manipulações do que adultos jovens ${ }^{3}$. De forma geral, esses estudos indicaram que idosos apresentam a capacidade de alterar a influência do estímulo sensorial se o mesmo apresentar uma variação abrupta na amplitude, mas mesmo assim essa variação ainda provoca uma maior influência em adultos idosos comparados com adultos jovens.

Os resultados deste estudo sugerem que uma possível explicação para tal diferença seria que idosos não conseguem discriminar tão bem pequenas variações do estímulo sensorial e alterar a relação entre a informação que esse estímulo propicia e a ação motora mais adequada para a situação. Novamente, essa alteração não envolve uma atuação consciente do indivíduo, conforme observado nas condições em que os idosos não foram informados sobre o movimento da sala e realizaram oscilação corporal correspondente. Essa diferença em alterar a relação entre o estímulo sensorial e a ação motora realizada, pelo menos quando ocorrem pequenas variações do estímulo sensorial, pode provocar situações de risco para idosos. Por exemplo, durante o caminhar, se ocorre uma pequena variação na superfície (calçada com uma parte mais elevada), essa variação pode passar sem ser notada e, conseqüentemente, a altura da passada não é aumentada, levando a um tropeço ou mesmo a uma queda.

A constatação de que idosos conseguem utilizar informação apresentada de forma extrínseca para alterar a influência do estímulo sensorial na ação motora indica que essa informação pode ser utilizada para compensar a diferença em obter as variações dos estímulos de forma implícita. Apesar de essa constatação ter sido observada em adultos jovens $^{22,23,31}$ e em crianças $^{24}$, não havia sido examinada em adultos idosos e constitui uma alternativa importante para compensar as alterações decorrentes do processo de envelhecimento. A apresentação de informação explícita possibilita que o sistema apresente alteração na relação entre informação sensorial e ação motora na mesma magnitude que a observada de forma implícita. Portanto, a compensação decorrente do uso de informação explicita possibilita a mesma performance na realização de ações motoras. O custo desse tipo de funcionamento é que o idoso tem de atuar de forma consciente, tendo de "prestar e/ou dirigir" atenção a aspectos apontados por outrem. Esta não é a forma mais eficiente de controlar os movimentos, mas constitui uma estratégia alternativa para essa população, dadas as alterações funcionais na relação entre informação sensorial e ação motora decorrente do envelhecimento.

Finalmente, mudanças ambientais também poderiam ser implementadas de forma a que as alterações em superfície, iluminação, textura, degraus etc. fossem destacadas. Para idosos - e para a população em geral - o destaque das mu- 
danças nas condições ambientais tem como função "direcionar", mesmo que explicitamente, a obtenção de informação sobre essas alterações. Assim, essas "dicas" ambientais para os idosos assumem uma importância ainda maior, pois o sistema já apresenta uma diferença na obtenção implícita de pequenas varia- ções ambientais. Portanto, direcionar o sistema para a obtenção dessas variações é crucial para a realização de ações motoras mais eficientes.

\section{REFERENCIAS}

1 Prioli AC, Cardozo AS, Freitas Júnior PB, Barela JA. Task demand effects on postural control in older adults. Hum Mov Sci. 2006;25:435-46.

2 Allison LK, Kiemel T, Jeka JJ. Multisensory reweighting of vision and touch is intact in healthy and fall-prone older adults. Exp Brain Res. 2006.

3 Prioli AC, Freitas Júnior PB, Barela JA. Physical activity and postural control in the elderly: coupling between visual information and body sway. Gerontology. 2005;51:145-8.

4 Ferraz MA, Barela JA, Pellegrini AM. Acoplamento sensório-motor no controle postural de indivíduos idosos fisicamente ativos e sedentários. Motriz. 2001;7(2):99-105.

5 McClenaghan BA, Williams HG, Dickerson J, Dowda M, Thombs L, Eleazer P. Spectral characteristics of ageing postural control. Gait Posture. 1996;4:112-21.

6 Wade MG, Lindquist R, Taylor JR, Treat-Jacobson D. Optical flow, spatial orientation, and the control of posture in the elderly. J Gerontol. 1995;50B(1):P51-P8.

7 Blaszczyk JW, Lowe DL, Hansen PD. Ranges of postural stability and their changes in the elderly. Gait Posture. 1994;2:11-7.

8 Amiridis IG, Hatzitaki V, Arabatzi F. Age-influence modifications of static postural control in humans. Neurosci Let. 2003;350:137-40.

9 Berger I, Chuzel M, Buisson G, Rougier P. Undisturbed upright stance control in the elderly: part 1. Age-related changes in undisturbed upright stance control. J Motor Behav. 2005;37(5):348-58.

10 Di Fabio RP, Emasithi A. Aging and the mechanisms underlying head and postural control during voluntary motion. Phys Ther. 1997;77(5):458-75.

11 Melzer I, Benjuya N, Kaplanski J. Postural stability in the elderly: a comparison between fallers and non-fallers. Age Ageing. 2004;33(6):602-7.

12 Lord SR, Menz HB, Tiedemann A. A physiological profile approach to falls risk assessment and prevention. Phys Ther. 2003;83(3):237-52.

13 Menz HB, Morris ME, Lord SR. Foot and ankle risk factors for falls in older people: a prospective study. J Gerontol. 2006;61(8):866-70.
14 Lord SR, Tiedemann A, Chapman k, Munro B, Murray SM, Sherrington C. The effects of an individualized fall prevention program on fall risk and falls in older people: a randomized, controlled trial. J Am Geriatr Soc. 2005;53:1296-304.

15 Horak FB. Postural orientation and equilibrium: what do we need to know about neural control of balance to prevent falls? Age Ageing. 2006;35:7-11.

16 Horak FB, Macpherson JM. Postural orientation and equilibrium. In: Rowell LB, Shepard JT, editors. Handbook of physiology. New York: Oxford University Press; 1996. p. 255-92.

17 Jeka J, Oie KS, Kiemel T. Multisensory information for human postural control: integrating touch and vision. Exp Brain Res. 2000;134:107-25.

18 Barela JA. Estratégias de controle em movimentos complexos: ciclo percepção-ação no controle postural. Rev Paul Educ Fis. 2000;(supl.3):79-88.

19 Grenne HA, Madden DJ. Adult age differences in visual acuity, stereopsis and contrast sensitivity. Am J Optom Physiol Opt. 1987;64:749-53.

20 Verrillo RT, Verrillo V. Sensory and perceptual performance. In: Charness N, editor. Ageing and human performance. New York: J Wiley \& Sons; 1985. p.1-46.

21 Polastri PF, Barela AMF, Barela JA. Controle postural em idosos: relacionamento entre informação visual e oscilação corporal. In: IX Congresso Brasileiro de Biomecânica, 2001; Gramado, RS. Anais. Porto Alegre: Escola de Educação Física da UFRGS; 2001. v.2, p.132-7.

22 Freitas Júnior PB, Barela JA. Postural control as a function of self-and object-motion perception. Neurosci Let. 2004;369:64-8.

23 Stoffregen TA, Hove P, Schmit J, Bardy BG. Voluntary and involuntary postural responses to imposed optic flow. Motor Control. 2006;10:24-33.

24 Perotti Jr. A, Barela JA, Polastri PF, Tani G. Influência de diferentes informações comportamentais na dinâmica intrínseca entre informação visual e oscilação corporal. Braz J Motor Behav. 2007;2(1):40-50.

25 Barela AM, Barela, JA, Rinaldi, NM, Toledo DR. Influence of imposed optic flow characteristics and intention on postural responses. Motor Control. 2009;13:1-12. 


\section{Referências (cont.)}

26 Jouen F, Lepecq JC, Gapenne O, Bertenthal BI. Optic flow sensitivity in neonates. Infant Behav Dev. 2000;23:271-84.

27 Barela JA, Godoi D, Freitas Junior P, Polastri PF. Visual information and body sway coupling in infants during sitting acquisition. Infant Behav Dev. 2000;23:285-97.

28 Barela JA. Adapted physical activity in promoting infant motor development. Rev Sobama. 2007;12(1):S42-7.
29 Carver S, Kiemel T, Jeka JJ. Modeling the dynamics of sensory reweighting. Biol Cyber. 2006;95(2):123-34.

30 Oie KS, Kiemel T, Barela JA, Jeka JJ. The dynamics of sensory reweighting: a temporal asymmetry. Gait Posture. 2005;21(1):S29.

31 Barela AMF, Barela JA, Rinaldi NM, Toledo DR. The influence of imposed optic flow characteristics and intention on postural responses. Motor Control. 2007. 